\title{
Serum (1->3)- B -D-Glucan Assay for Diagnosis of Pneumocystis Pneumonia in Immunocompromised Patients
}

\author{
Li-Chao Fan', Jie-Ming $\mathbf{Q}{ }^{2}$, and Jin-Fu Xü*
}

${ }^{1}$ Department of Respiratory Medicine, Shanghai Pulmonary Hospital, Tongji University School of Medicine, Shanghai, 200433, China

${ }^{2}$ Department of Respiratory Medicine, Huadong Hospital, Fudan University, Shanghai, 200040, China

Pneumocystis Pneumonia (PCP) is one of the most prevalent infections in Human Immunodeficiency Virus (HIV)-Infected Patients [1]. PCP also occurs in non-HIV patients who receiving transplantation, immunosuppressive therapy and anti-tumor chemotherapeutic agents [2]. The diagnosis of PCP is usually based on the microscopic detection of cysts by certain stain in respiratory specimens among patients at risk with fever, progressive dyspnea, nonproductive cough and relevant pulmonary infiltrates. However, the methods are limited to the quality of the respiratory specimens and the skill of the observer. Novel tests with greater sensitivity, such as Polymerase Chain Reaction (PCR) of respiratory samples, are potentially promising, but not yet widely available [3]. Emerging data from recent years have pointed to serum $(1->3)-\beta$-D-Glucan (BG) measurement as a promising tool for the diagnosis of PCP [4-6].

The peripheral measurement of (1->3)- $\beta$-D-glucan is based on detection of $\beta$-glucan, a polysaccharide present in the wall of Pneumocystis cysts and in other fungi $[7,8]$. The tests commercially available for the detection of BG are varied like the Fungitell assay (Associates of Cape Cod Inc., East Falmouth, MA, USA), the Fungitec G-test MK assay, the G-test assay (both are developed by Seikagaku Corp, Tokyo, Japan), b-glucan test Wako (Wako Pure Chemical Industries Ltd, Osaka, Japan) and so on. Among them Fungitell assay is approved in 2003 by the U.S. Food and Drug Administration for the presumptive diagnosis of invasive fungal infection. BG value of $80 \mathrm{pg} /$ $\mathrm{ml}$ was considered positive according to the manufacturer's indications. The accuracy of BG maybe varies owing to the cutoff value of BG.

A meta-analysis made of fourteen studies reported that the average (95\% confidence interval) sensitivity and specificity of BG were $94.8 \%(90.8-97.1 \%)$ and $86.3 \%(81.7-89.9 \%)$, respectively. The positive and negative likelihood ratios were $6.9(5.1-9.3)$ and 0.06 (0.03-0.11), respectively. The area under the HSROC curve was 0.965 (0.945-0.978) [9]. The pooled specificity was moderate for PCP because the BG assay could be positive for various fungal infections and the presence of factors such as use of intravenous amoxicillin-clavulanic acid, treatment of patients with immunological preparations (albumins or globulins), use of cellulose membranes and filters made from cellulose in hemodialysis, and use of cotton gauze swabs/packs/pads and sponges during surgery [10]. If the aim is to increase the specificity of the test, slightly higher cut-off level than the one used in invasive fungal infections may potentially provide higher specificity, without unacceptable loss in sensitivity [4].

One report shows an excellent diagnostic performance, with a sensitivity of $98 \%$ and a specificity of $94 \%$, the positive predictive value was only $64.7 \%$ and the negative predictive value is $99.8 \%$ [5]. The unsatisfactory PPV is possibly owing to affect by prevalence rate of local people, concurrent infection of other invasive fungi, intravenous injection of albumins or globulins, use of cellulose membranes and so on. So diagnosis of PCP should combine with clinical context. However, with a negative predictive value of $99.8 \%$, a BG level $\leq 85 \mathrm{pg} / \mathrm{mL}$ almost rules out PCP. Moreover, Serum BG could potentially be of value in differentiating between colonization and infection of PCP $[11,12]$.

The severity and mortality of PCP, as well as BG levels, have been reported to be lower in HIV-positive patients than in non-HIV patients $[13,14]$. However other studies didn't show significant difference between them $[5,6,15]$. Further evaluation is needed to develop this controversy.

As regard to the value of prognosis of BG, a study shows that decreasing BG levels show a good correlation (88\%) with a favorable treatment response [16]. This may assist in deciding whether to continue first-line therapy in cases of missing clinical improvement. Meanwhile, the kinetics of measured BG (Fungitell; Associates of Cape Cod) suggest that whereas increasing levels soon after treatment may not associated with treatment failure for b-d-glucan levels seldom return to the normal level within a 21-day treatment [17]. The increasing BG levels maybe the lysis of fungi after treatment.

In conclusion, the BG test is helpful for the diagnosis of PCP with a high accuracy, especially for patients with severe respiratory failure who are unable to undergo bronchoscopy and other invasive examinations owing to severe hypoxemia.

\section{Acknowledgment}

This work was supported by China Natural Science Foundation project (81170003) and Shanghai Young Medical Elite Project (XYQ2011006).

\section{Reference}

1. Morris A, Lundgren JD, Masur H, Walzer PD, Hanson DL, et al. (2004) Current epidemiology of Pneumocystis pneumonia. Emerg Infect Dis 10: 1713-1720.

2. Mansharamani NG, Garland R, Delaney D, Koziel H (2000) Management and outcome patterns for adult Pneumocystis carinii pneumonia, 1985 to 1995 comparison of HIV-associated cases to other immunocompromised states. Chest 118: 704-711.

3. Turner D, Schwarz Y, Yust I (2003) Induced sputum for diagnosing Pneumocystis carinii pneumonia in HIV patients: new data, new issues. Eur Respir J 21: 204 208

4. Desmet S, Van Wijngaerden E, Maertens J, Verhaegen J, Verbeken E, et al. (2009) Serum (1-3)-beta-D-glucan as a tool for diagnosis of Pneumocystis jirovecii pneumonia in patients with human immunodeficiency virus infection or hematological malignancy. J Clin Microbiol 47: 3871-3874.

5. Held J, Koch MS, Reischl U, Danner T, Serr A (2011) Serum (1 $\rightarrow$ 3)-B-D-glucan measurement as an early indicator of Pneumocystis jirovecii pneumonia and evaluation of its prognostic value. Clin Microbiol Infect 17: 595-602.

6. Watanabe T, Yasuoka A, Tanuma J, Yazaki H, Honda H, et al. (2009) Serum (1-->3) beta-D-glucan as a noninvasive adjunct marker for the diagnosis of Pneumocystis pneumonia in patients with AIDS. Clin Infect Dis 49: 1128-1131.

${ }^{*}$ Corresponding author: Jin-Fu Xu, Department of Respiratory Medicine Shanghai Pulmonary Hospital, Tongji University School of Medicine, Shanghai, 200433, China, E-mail: jfxu@ymail.com

Received November 26, 2012; Accepted November 27, 2012; Published November 28, 2012

Citation: Fan LC, Qu JM, Xu JF (2012) Serum (1->3)-B-D-Glucan Assay for Diagnosis of Pneumocystis Pneumonia in Immunocompromised Patients. Pulmon Resp Med 2:e121. doi:10.4172/2161-105X.1000e121

Copyright: (c) 2012 Fan LC. This is an open-access article distributed under the terms of the Creative Commons Attribution License, which permits unrestricted use, distribution, and reproduction in any medium, provided the original author and source are credited. 
Citation: Fan LC, Qu JM, Xu JF (2012) Serum (1->3)-B-D-Glucan Assay for Diagnosis of Pneumocystis Pneumonia in Immunocompromised Patients. J Pulmon Resp Med 2:e121. doi:10.4172/2161-105X.1000e121

7. Finkelman MA (2010) Pneumocystis jirovecii infection: Cell wall (1 $\rightarrow 3)-\beta-D-$ glucan biology and diagnostic utility. Crit Rev Microbiol 36: 271-281.

8. Matsumoto $\mathrm{Y}$, Matsuda S, Tegoshi T (1989) Yeast glucan in the cyst wall of Pneumocystis carinii. J Protozool 36: 21S-22S

9. Karageorgopoulos DE, Qu JM, Korbila IP, Zhu YG, Vasileiou VA, et al. (2011) Accuracy of $\beta$-d-glucan for the diagnosis of Pneumocystis jirovecii pneumonia: a meta-analysis. Clin Microbiol Infect.

10. Carmona EM, Limper AH (2011) Update on the diagnosis and treatment of Pneumocystis pneumonia. Ther Adv Respir Dis 5: 41-59.

11. Matsumura $Y$, Ito $Y$, linuma $Y$, Yasuma K, Yamamoto M, et al. (2012) Quantitative real-time PCR and the $(1 \rightarrow 3)-\beta$-D-glucan assay for differentiation between Pneumocystis jirovecii pneumonia and colonization. Clin Microbiol Infect 18 591-597.

12. Shimizu Y, Sunaga N, Dobashi K, Fueki M, Fueki N, et al. (2009) Serum markers in interstitial pneumonia with and without Pneumocystis jirovecii colonization: a prospective study. BMC Infect Dis 9: 47.
13. Fujii T, Nakamura T, Iwamoto A (2007) Pneumocystis pneumonia in patients with HIV infection: clinical manifestations, laboratory findings, and radiological features. J Infect Chemother 13: 1-7.

14. Nakamura H, Tateyama M, Tasato D, Haranaga S, Yara S, et al. (2009) Clinical utility of serum beta-D-glucan and KL-6 levels in Pneumocystis jiroveci pneumonia. Intern Med 48: 195-202.

15. Onishi A, Sugiyama D, Kogata Y, Saegusa J, Sugimoto T, et al. (2012) Diagnostic accuracy of serum 1,3- $\beta$-D-glucan for pneumocystis jirovec pneumonia, invasive candidiasis, and invasive aspergillosis: systematic review and meta-analysis. J Clin Microbiol 50: 7-15.

16. Held J, Wagner D (2011) $\beta$-d-Glucan kinetics for the assessment of treatment response in Pneumocystis jirovecii pneumonia. Clin Microbiol Infect 17: 11181122.

17. Del Palacio A, Llenas-García J, Soledad Cuétara M, Pulido F, Rubio R, et al. (2010) Serum (1-->3) beta-D-Glucan as a noninvasive adjunct marker for the diagnosis and follow-up of pneumocystis jiroveci pneumonia in patients with HIV infection. Clin Infect Dis 50: 451-452. 